\title{
PEPTÍDEOS DE CONFORMAÇÃO RESTRITA INDUZIDA PELA INCORPORAÇÃO DE UNIDADES (AZA)LACTÂMICAS
}

\author{
Adriana Raffin Pohlmann \\ Departamento de Química Orgânica - Instituto de Química - UFRGS - CP 15003 - 91501-970 - Porto Alegre - RS \\ Jean-Charles Quirion \\ Laboratoire d'Hétérochimie Organique - IRCOF - Institut National des Sciences Appliquées de Rouen - França \\ Dominique Guillaume e Henri-Philippe Husson \\ Laboratoire de Chimie Thérapeutique - CNRS 1310 - Faculté des Sciences Pharmaceutiques et Biologiques - Université René \\ Descartes - Paris V
}

Recebido em 21/8/98; aceito em 29/1/99

\begin{abstract}
CONFORMATIONALLY CONSTRAINED INDUCED PEPTIDES CONTAINING (AZA)LACTAM UNITS. Conformational constraint is an approach which can be used to restrict the flexibility of peptide molecules and to provide information on the topographical requirements of receptors. The incorporation of conformationally constrained units in a peptide can lead to peptide analogues that present numerous advantages such as the potentialization of the pharmacological activity and the decrease of enzymatic degradation. This review discusses the peptide analogues containing a lactam or azalactam unit in their sequences. Of particular interest has been the replacement of a dipeptide motif in a peptide that simulates a $\beta$-turn.
\end{abstract}

Keywords: peptide analogues; conformational constraint; lactam; azalactam; structure-activity.

\section{INTRODUÇÃO}

As proteínas e os peptídeos, cadeias de aminoácidos, são componentes essenciais no organismo. As proteínas possuem igualmente as funções de constituintes dos tecidos e de agentes biocatalíticos e hormonais ${ }^{1}$. Os peptídeos, por sua vez, atuam como hormônios, neurotransmissores e/ou neuromoduladores, apresentando, portanto, uma grande diversidade de atividades biológicas e numerosas propriedades terapêuticas ${ }^{1}$. Entretanto, suas utilizações como agentes terapêuticos são limitadas essencialmente por sua baixa biodisponibilidade oral e/ou características farmacodinâmicas, e ainda pela sensibilidade às peptidases ${ }^{1}$.

A flexibilidade conformacional dos peptídeos é uma vantagem, mas ao mesmo tempo pode ser considerada um fator limitante de sua atividade ${ }^{2-4}$. Esta flexibilidade está ligada às possibilidades de rotação das ligações simples de cada resíduo de aminoácido da cadeia peptídica (Figura 1). Os ângulos de torsão $\phi, \psi$ e $\omega$, estabelecidos pelos diedros $\mathrm{C}_{i-1}-\mathrm{N}_{i^{-}} \mathrm{C}_{\alpha^{-}} \mathrm{C}_{i}, \mathrm{~N}_{i^{-}}$ $\mathrm{C}_{\alpha}-\mathrm{C}_{i}-\mathrm{N}_{i+1}$ e pela ligação amida, apresentam valores característicos em sequiências polipeptídicas naturais, cujas dobras são estabilizadas por pontes de hidrogênio. Cada combinação de valores dos ângulos $\phi, \psi$ e $\omega$ produz elementos de estrutura secundária, classificados segundo três tipos: hélice $\alpha$, folhas pregueadas $\beta$ e dobras ${ }^{2,5}$. Segundo os valores de $\phi, \psi$ e $\omega$, as dobras podem ser classificadas como $\beta \mathrm{I}, \beta \mathrm{I}$ ', $\beta \mathrm{II}, \beta \mathrm{II}$ ', ou ainda como $\gamma$ e $\gamma$ inversa, indicadas na Figura 2 e Tabela 1 .

$\mathrm{O}$ equilíbrio entre as possíveis conformações das sequiências peptídicas leva a confôrmeros de diferentes estruturas secundárias. Os confôrmeros geralmente não são isoláveis e por consequência suas atividades biológicas não podem ser testadas separadamente $^{3,6}$. Uma alternativa de investigação para a determinação da relação entre a estrutura tridimensional e a atividade biológica foi proposta com base no modelo de interação hormônio (ou neurotransmissor) e seu receptor ${ }^{6,7}$. Segundo este modelo, o ligante ocasiona uma mudança de conformação do receptor, ativando uma resposta biológica ${ }^{6,8}$. Para um peptídeo natural, a região ativa compreende uma pequena porção do peptídeo (quatro a cinco resíduos), sendo o restante da molécula necessário para favorecer a estrutura secundária da seqüência ativa ${ }^{6}$. A restrição conformacional pode ser utilizada como abordagem para desenvolver estudos de determinação da relação estrutura - atividade. A fixação de uma ou várias zonas do ligante minimiza o número de conformações da sequiência peptídica e, por conseqüência, limita o grau de liberdade dos ângulos $\phi$ e $\psi$.

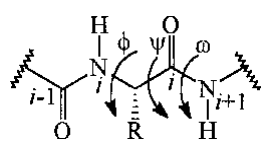

Figura 1. Ângulos de Tosão $\phi$, $\psi$ et $\omega$.

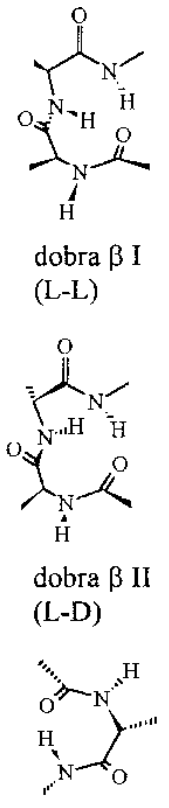

dobra $\gamma$

(D)

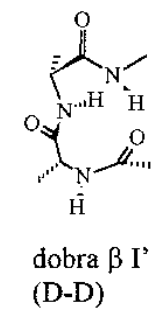<smiles>CNC(=O)C(C)NC(=O)NC(C)C</smiles>

dobra $\beta$ II' (D-L)<smiles>CCC(NC(C)=O)C(=O)NC</smiles>

dobra $\gamma$ inversa

(L)
Figura 2. Representação das dobras $\beta$ e $\gamma^{2,5}$. 
Tabela 1. Valores dos ângulos de diedro das dobras $\beta$ e $\gamma$.

\begin{tabular}{ccccc}
\hline $\begin{array}{l}\beta \text {-loop } \\
\text { reverso }\end{array}$ & $\phi_{\mathrm{i}+1}$ & $\psi_{\mathrm{i}+1}$ & $\phi_{\mathrm{i}+2}$ & $\psi_{\mathrm{i}+2}$ \\
\hline tipo I & -60 & -30 & -90 & 0 \\
tipo I' & +60 & +30 & +90 & 0 \\
tipo II & -60 & +120 & +80 & 0 \\
tipo II' & +60 & -120 & -80 & 0 \\
& & & & \\
& $\psi_{\mathrm{i}}$ & $\phi_{\mathrm{i}+1}$ & $\psi_{\mathrm{i}+1}$ & $\phi_{\mathrm{i}+2}$ \\
\hline$\gamma$-loop & +120 & -65 & +80 & -120 \\
reverso & & & & \\
\hline
\end{tabular}

A diminuição do número de conformações pode ser obtida pela incorporação de restritores conformacionais. Existem vários métodos para restringir a rotação dos diedros $\phi, \psi$ e $\omega$. Os mais comumente utilizados são baseados na formação de cadeias $\mathrm{N}$-metiladas ou de pontes dissulfeto, na incorporação de ligações duplas ou da prolina, ou ainda na substituição dos constituintes da seqüência peptídica por outros grupamentos ${ }^{1-3}$. Outra alternativa é realizar diferentes tipos de ciclização entre os resíduos, como mostra a Figura 3, por exemplo: entre dois aminoácidos consecutivos $i$ e $i+1$, ou entre resíduos alternados $i$ e $i+2^{3}$. As ciclizações podem ser realizadas entre duas cadeias laterais, entre a cadeia lateral e o esqueleto peptídico, bem como por conexão entre os átomos do esqueleto peptídico ${ }^{3}$.

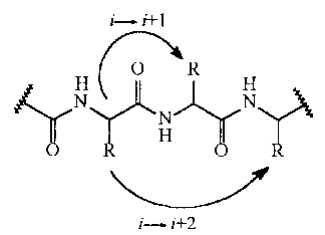

Figura 3. Conexões para a civilização de peptídeos ${ }^{3}$.

A introdução de restritores conformacionais pode apresentar numerosas vantagens como a potencialização da atividade farmacológica por estabilização de um confôrmero ${ }^{9 a}$, a diminuição da degradação enzimática pela eliminação das formas metabolizáveis ${ }^{9 a}$, o aumento da seletividade pela diminuição dos confôrmeros bioativos responsáveis por efeitos secundários e, enfim, fornecer inúmeras informações sobre a conformação biologicamente ativa de um peptídeo em relação a um receptor específico ${ }^{9}$.

\section{ANÁLOGOS PEPTÍDICOS (AZA)LACTÂMICOS}

A existência de ligações amida nos peptídeos levou ao desenvolvimento de uma estratégia de restrição conformacional baseada na incorporação de lactamas em seqüências ativas. A idéia surgiu da concepção de um restritor conformacional, que permitisse a diminuição da rotação do ângulo de torção $\mathrm{N}_{i}-\mathrm{C}_{\alpha}-\mathrm{C}_{i}-\mathrm{N}_{i+1}$ e conseqüentemente da ligação amida de uma seqüência peptídica 9 .

Freidinger e colaboradores ${ }^{9 a, b}$ foram pioneiros na utilização de um ciclo lactâmico para obter um análogo peptídico de conformação restrita. Desde então, vários métodos de síntese de restritores do tipo lactâmico foram desenvolvidos para produzir anéis simples, condensados ou macrocíclicos ${ }^{1,3,10}$. Muitos análogos peptídicos foram preparados, a partir da aplicação desses métodos de síntese em combinação com os métodos de condensação peptídica, com objetivos farmacológicos diferenciados ${ }^{1-3,10}$.

Inúmeros exemplos de neuropeptídeos (hormônios, neurotransmissores e neuromoduladores) e inibidores de enzimas junto com diversas lactamas foram utilizados para produzir análogos peptídicos e não-peptídicos de conformação restrita. Devido a grande complexidade para abranger todos os aspectos que correlacionam estes temas, esta revisão limitar-se-á a um único aspecto da restrição conformacional de cadeias peptídicas pela incorporação de lactamas e azalactamas em suas seqüências. Os exemplos de inclusão de unidades (aza)lactâmicas não-peptídicas ou de formação de macrociclos não serão descritos.

\section{Análogo do hormônio de liberação da luteína}

Os primeiros análogos peptídicos de conformação restrita, incorporando um ciclo lactâmico, foram descritos por Freidinger e colaboradores ${ }^{9 a, b}$, em 1980 . O análogo restrito $\mathbf{1}$ do hormônio de liberação da luteína (LH-RH, 2, Figura 4) foi preparado pela incorporação de uma unidade dipeptídica restrita no lugar dos resíduos Gly-Leu do LH-RH. A conformação da unidade dipeptídica é limitada pela presença de uma $\gamma$-lactama, onde uma ponte etilênica une o nitrogênio do resíduo leucina ao carbono $\alpha$ da glicina.

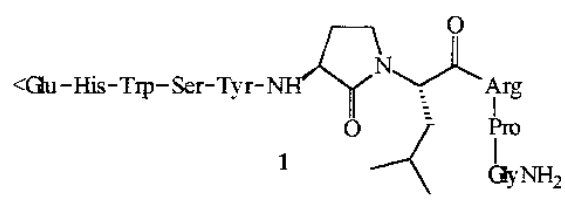

$<$ Glu-His-Trp-Ser-Tyr-Gly-Leu-Arg-Pro-Gly- $\mathrm{NH}_{2}$

\section{LH-RH, 2}

Figura 4. Análogo restrito do homônio de liberação da luteína ${ }^{9 a}$.

O análogo 1 apresentou uma restrição local de dobra $\beta$ em torno da unidade restritora e os resultados dos ensaios farmacológicos mostraram que 1 é mais ativo que o LH-RH, 2. Estes resultados permitiram a proposição de uma dobra $\beta$ (em torno dos resíduos Gly-Leu) como conformação ativa do hormônio natural 2.

\section{Análogos do tripeptídeo Pro-Leu-GlyNH}

O estudo da relação estrutura - atividade do tripeptídeo LPro-L-Leu-GlyNH $\mathrm{N}_{2}$ (PLG, 3, Figura 5) foi realizado a partir da preparação de análogos peptídicos contendo $\gamma$ - e $\delta$-lactamas ${ }^{11-15}$. O PLG é um tripeptídeo endógeno que possui vários tipos de atividades sobre o sistema nervoso central. O PLG teria um efeito direto na modulação do sistema dopaminérgico, capaz de potencializar os efeitos da L-Dopa e da apomorfina.

A primeira série de análogos 4-6 foi preparada substituindo os resíduos Leu-GlyNH${ }_{2}$ de 3 por unidades restritoras dipeptídicas $\gamma$ - e $\delta$-lactâmicas ${ }^{12,15}$, nas quais o átomo de nitrogênio da glicina está ligado à cadeia lateral da leucina. $\mathrm{Na}$ segunda série, o resíduo glicinamida do PLG foi substituído por heterociclos nitrogenados ${ }^{13}$, fornecendo os análogos 9-11. Os derivados 9 e $\mathbf{1 0}$ possuem ciclos lactâmicos, onde o grupamento $\mathrm{NH}_{2}$ da glicinamida é ligado ao carbono $\alpha$ deste resíduo por uma ponte etilênica ou por uma ponte propilênica. No caso do derivado 11, o heterociclo é uma 2-oxopiperazina, onde os nitrogênios do resíduo glicinamida estão ligados por uma ponte etilênica.

O composto 5 demonstrou uma atividade moduladora significativa em relação aos outros derivados. Este resultado foi justificado pela hipótese de que os isômeros de configuração $(R)$ mimetizam uma dobra $\beta$ II, enquanto que os isômeros de configuração $(S)$, mimetizam uma dobra $\beta$ II'. A conformação bioativa do PLG seria do tipo dobra $\beta$ II.

Baseado nesta hipótese, vários análogos restritos do tripeptídeo com este tipo de dobra foram preparados para dar continuidade aos estudos da relação estrutura - atividade $^{16}$. Os compostos 
<smiles>CC(C)C[C@H](NC(=O)[C@@H]1CCCN1)C(=O)NCC(N)=O</smiles><smiles>NC(=O)CN1CCC(=NC(=O)C2CCCN2[Al])C1=O</smiles>

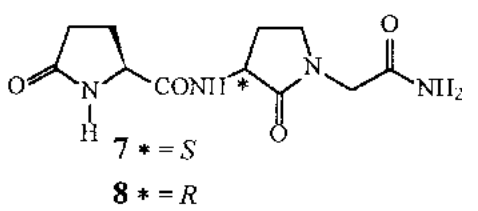<smiles>[R]CNC1CCNC1=O</smiles>

Figura 5. PLG 3 e análagos restritos ${ }^{12,13}$.

12-17 (Figura 6) foram obtidos substituindo-se a $\gamma$-lactama de 5 por outras lactamas em função da variação do ângulo de torsão $\psi_{2}$.

As diferentes unidades restritoras foram posicionadas de modo a limitar a conformação em torno dos resíduos Leu$\mathrm{GlyNH}_{2}$ do PLG, ligando o nitrogênio do resíduo glicinamida a diferentes átomos da cadeia lateral da leucina. Os análogos 12-15 e 17, onde os valores do ângulo $\psi_{2}$ são próximos aos valores observados para uma dobra $\beta$ II, mostraram-se ativos, confirmando a hipótese de que este tipo de dobra é a conformação bioativa do PLG. Com o objetivo de obter compostos ainda mais rígidos, os análogos lactamtiazolidina 18 e 19 foram preparados ${ }^{17}$ (Figura 6), e os resultados dos ensaios farmacológicos mostraram que são mais ativos que o PLG.

Pode-se encontrar na classe das dicetopiperazinas o ciclo[LeuGly], um análogo restrito do PLG. O ciclo[Leu-Gly] possui efeitos opostos sobre os receptores dopaminérgicos conforme a região cerebral onde estes se encontram ${ }^{18-20}$.

As dicetopiperazinas, dipeptídeos cíclicos endógenos, são substâncias de grande interesse biológico ${ }^{21}$, encontradas nos reinos animal e vegetal. Cabe salientar que estas substâncias são os derivados peptídicos mais simples encontrados na natureza, dos quais vários exemplos provém de subprodutos da fermentação ou do cozimento dos alimentos, em particular da carne. Antes mesmo de serem conhecidas, algumas dicetopiperazinas sintéticas haviam sido obtidas em 1888. Esses compostos apresentam várias atividades fisiológicas e farmacológicas em mamíferos ${ }^{21}$. Os dipeptídeos cíclicos, adequadamente substituídos em suas cadeias laterais, fornecem estruturas restritas com utilidade em estudos de reconhecimento molecular e de determinação conformacional ${ }^{22}$.

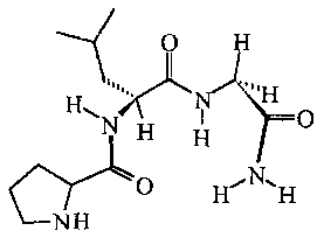

3

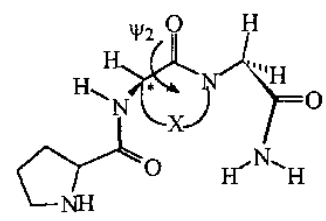

$\begin{array}{rll}5 & \mathrm{X}=\mathrm{CH}_{2} \mathrm{CH}_{2} & *=R \\ 12 & \mathrm{X}=\mathrm{CH}_{2} & *=R \\ 13 & \mathrm{X}=\mathrm{CH}_{2} \mathrm{CO} & *=R \\ 14 & \mathrm{X}=\mathrm{CH}_{2} \mathrm{O} & *=R \\ 15 & \mathrm{X}=\left(\mathrm{CH}_{2}\right)_{3} & *=R \\ 16 & \mathrm{X}=\left(\mathrm{CH}_{2}\right)_{4} & *=R \\ 17 & \mathrm{X}=\left(\mathrm{CH}_{2}\right)_{4} & *=S\end{array}$

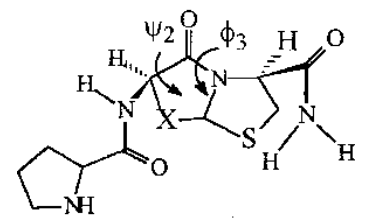

$18 \mathrm{X}=\mathrm{CH}_{2}$

$19 \mathrm{X}=\mathrm{CH}_{2} \mathrm{CH}_{2}$

Figura 6. Análogos restritos do PLG $3^{21,22}$.

Assim como os dipeptídeos cíclicos, as $\gamma$ - e $\delta$-lactamas, bem como o sistema biciclo 6,5-lactamtiazolidina foram propostos por outros autores e incorporados em peptídeos, gerando análogos das encefalinas, do LH-RH, da somatostatina, da gramicidina $\mathrm{S}$ e do fator de liberação do hormônio de crescimento $^{23-27}$. Outros polipeptídeos cíclicos, além da gramicidina $S$, foram alvo de estudos conformacionais. Um exemplo é a ciclosporina $\mathrm{A}$, cujos análogos $\gamma$ - e $\delta$-lactâmicos foram preparados. Entretanto, estes derivados restritos apresentaram bai$\mathrm{xa}$ atividade imunossupressora ${ }^{28,29}$.

\section{Análogos da substância $\mathbf{P}$}

Outros tipos de unidades de restrição podem mimetizar uma dobra $\beta$. As espiro[4.4]lactamas foram descritas como estruturas capazes de estabilizar uma dobra $\beta$ II, uma vez que sua configuração é $R$, e uma dobra $\beta$ II' quando sua configuração é $S^{30-35}$.

A substância $\mathrm{P}$ sinaliza a dor e a agressão física ao corpo ${ }^{36}$. As espiro[4.4]lactamas foram utilizadas para preparar uma série de análogos da substância P (20, Figura 7), desenvolvidos tanto para estudar o modo de ação deste neuropeptídeo em algumas doenças, como para caracterizar seus receptores. Os receptores das taquicininas de mamíferos [substância P (SP), neurocinina A (NKA) e neurocinina $B(\mathrm{NKB})$ ] foram classificados em três subgrupos de receptores (NK-1, NK-2 e NK-3) com base na atividade de agonistas seletivos $^{32,35 a}$. O análogo $\delta$-aminovalerila da SP ([Ava $\left.{ }^{6}\right]$ SP, 21) foi escolhido como agonista de referência para a realização dos estudos da determinação da relação estrutura - atividade 

Arg $^{1}$-Pro ${ }^{2}-$ Lys $^{3}$-Pro
4
Substância P,
Sub
Ava ${ }^{6}$-Phe-Phe-Gly-Leu-Met- $\mathrm{NH}_{2}$ [Ava $\left.{ }^{6}\right]-$ SP, 21

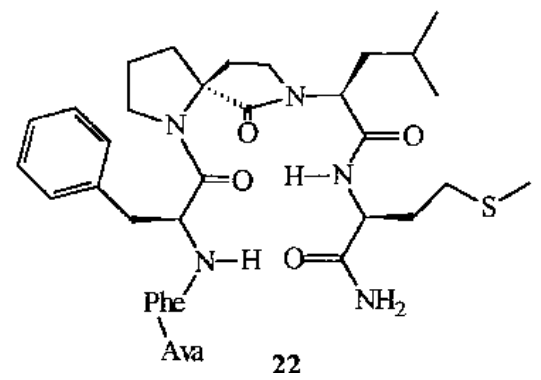

Figura 7. Substância P 20, $\left[A v a^{6}\right]-S P 21$ e análogo espirolactâmico $22^{35 a}$.

sobre os receptores NK-1 e NK-2. O derivado 22 foi preparado a partir da substituição do resíduo glicina de [Ava $\left.{ }^{6}\right]-S P$ (21) pela unidade de restrição espiro[4.4]lactama. Os resultados demonstraram que o composto 22 não possui ação como agonista, entretanto, sua afinidade pelo receptor NK-1 foi suficiente para caracterizar uma ação como antagonista competitivo ${ }^{35 a}$.

Os derivados 23 e 24 (Figura 8) foram preparados a partir dos estudos sobre os agonistas do receptor NK-2 $2^{35 b}$. O composto $\mathbf{2 3}$ foi gerado pela incorporação de uma $\gamma$-lactama no lugar dos resíduos Gly-Leu da porção C-terminal de [Lys $\left.{ }^{3}\right]$ NKA (25), análogo da neurocinina A (26), e o derivado 24 pela inserção de um biciclo[3.3.0] substituindo também os resíduos Gly-Leu, desta vez, do análogo [Ava $\left.{ }^{6}\right]-S P, 21$.

Em 1996, foi descrito um método de síntese para obter-se uma unidade de restrição do tipo 2-oxopiperazina condensada (biciclo[4.3.0]), ligando-se os átomos $\mathrm{N}_{i}, \mathrm{~N}_{i+1}$ e $\mathrm{C}_{\beta i+1}$ de um fragmento peptídico ${ }^{37}$.

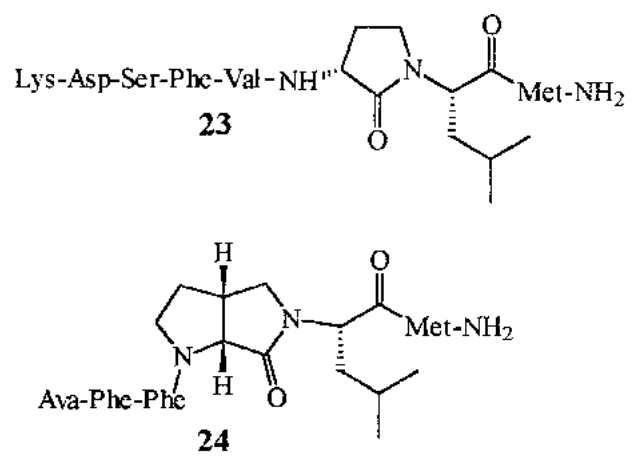

$$
\begin{gathered}
\text { Lys }^{3}-\text { Aspy-Ser-Phe-Val-Gly-Leu-Met-NH } \\
\text { [Lys } \left.{ }^{3}\right]-\mathrm{NKA}, 25
\end{gathered}
$$

\section{$\mathrm{His}^{1}-\mathrm{Lys}^{2}-\mathrm{Thr}^{3}$-Asp-Ser-Phe-Val-Gly-Leu-Met-NH neurocinina A, 26}

Figura 8. Análogos dos agonistas do receptor $N K-2^{35 b}$.

\section{Inibidores da renina}

As lactamas foram igualmente utilizadas como unidades de restrição nas séries de análogos dos inibidores do sistema renina-angiotensina-aldosterona. Este sistema é uma cascata proteolítica presente na circulação, bem como em outros tecidos, com finalidade de prover um mecanismo de urgência no caso de hipovolemia (ex: hemorragia $)^{36}$. A renina provoca a lise do angiotensinogênio, fornecendo a angiotensina I. Esta é transformada em angiotensina II pela enzima conversora da angiotensina (ECA). A angiotensina II, um potente vasoconstritor, estimula a liberação de aldosterona que induz a retenção do sódio e da água contribuindo ao aumento da pressão sanguínea ${ }^{36}$. No caso da necessidade do controle da hipertensão, a interrupção dessa cascata pode ser efetuada em dois níveis: pela inibição da renina ou pela inibição da $\mathrm{ECA}^{38,39}$.

$\mathrm{O}$ angiotensinogênio 27 (Figura 9) é hidrolisado pela renina entre os resíduos $\mathrm{Leu}^{10}-\mathrm{Val}^{11}$. Uma classe de inibidores potentes da renina foi desenvolvida pela obtenção de pequenos peptídeos, onde os resíduos 10 e 11 são substituídos por carbonos tetraédricos não hidrolisáveis. O carbono tetraédrico no lugar da carbonila mimetiza a geometria do estado de transição da hidrólise de amidas. Como exemplos desta classe de inibidores pode-se citar os análogos 28 e 29 , incorporando a estatina. Uma série de derivados restritos (30-32) destes inibidores foi desenvolvida pela inserção de uma $\gamma$-lactama nos sítios P2-P3 dos peptídeos com a intenção de minimizar confôrmeros metabolizáveis dos derivados estatina ${ }^{38}$. O peptídeo restrito 30 demonstrou baixa atividade inibidora, no entanto, os epímeros 31 e os $\mathbf{3 2}$ apresentaram uma inibição de mesma ordem que as referência não restritas.

Os compostos do tipo 33-35 ${ }^{39}$ (Figura 10), entre outros, foram preparados pela incorporação de $\gamma$-, $\delta$ - e $\varepsilon$-lactamas substituindo a estatina dos inibidores do tipo 28. Os resultados dos ensaios com o derivado mais potente $\mathbf{3 6}$ sugerem que a porção hidrofóbica do anel $\gamma$-lactâmico pode mimetizar a cadeia lateral de P1' do angiotensinogênio.

\section{Inibidores da enzima conversora da angiotensina}

O desenvolvimento dos inibidores da ECA teve início a partir dos estudos de relação estrutura - atividade de diversos substratos. Os derivados do dipeptídeo Ala-Pro se mostraram bons substratos para a enzima e alguns análogos simples contendo a prolina na porção C-terminal se mostraram bons inibidores ${ }^{40-43}$. Vários dipeptídeos restritos foram utilizados na elaboração de análogos de inibidores da ECA. Com base na conformação e nas propriedades inibidoras do captopril 37 e do enalapril 38 (Figura 11), vários inibidores de conformação restrita, contendo uma lactama do tipo monocíclica ${ }^{43,44}$, bicíclica ${ }^{41,45-47}$ ou tricíclica ${ }^{48-50}$ foram sintetizados como, por exemplo, os compostos 39-41.

\section{Inibidores duplos de metaloproteases}

Em 1993, a unidade de restrição do tipo 1,4-oxazepínico foi produzida com o objetivo de se obter análogos de inibidores da ECA, bem como da atriopeptidase (NEP) ${ }^{51}$. Em seguida, várias séries de derivados do tipo 42 (Figura 12), análogos dos inibidores de metaloproteases $(\text { ECA/NEP) })^{52-54}$ 


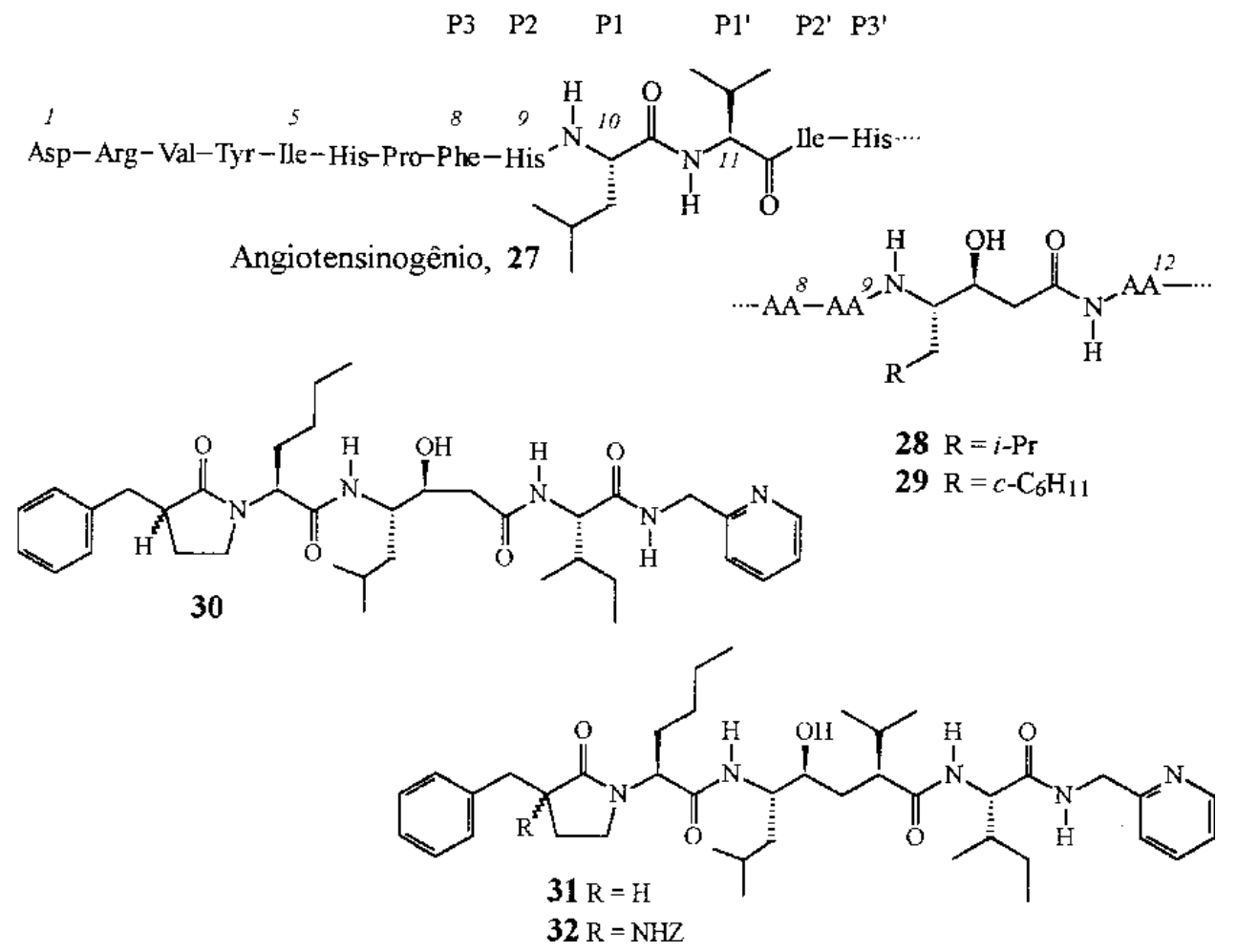

Figura 9. Angiotensinogênio 27 e inibidores da renina $28-32^{38}$.<smiles>CNC(CC1CCCCC1)N(NC)C(O)C1CCN(C)C1=O</smiles><smiles>CN1C(=O)[C@H]([C@H](O)[C@H](CC2CCCCC2)N[CH]C(=O)c2ccccc2)CC1(C)C</smiles>

Figura 10. Análogos restritos dos inibidores da renina ${ }^{39}$.

foram desenvolvidas incorporando núcleos benzoxazepinônicos, benzazepinônicos e benzatiazepinônicos.

Duas séries de análogos azepinona (mono- e bicíclicos) foram descritas $^{55}$ (Figura 13) a partir de estudos da relação estrutura atividade dos inibidores de metaloprotease ${ }^{56}$. Os derivados 43 e 44, os mais ativos deste estudo, foram obtidos pela incorporação de uma ponte etilênica entre o grupamento metila da alanina e a posição C-5 do núcleo prolina do análogo inativo 45.

\section{Inibidores da elastase de leucócitos humanos}

Uma série de derivados peptídicos, análogos dos inibidores da elastase de leucócitos humanos, foi produzida pela inclusão de uma lactama, fixando a ligação amida trans e restringindo os valores do ângulo $\psi$ dos inibidores do tipo $\mathbf{4 6}{ }^{57}$ (Figura 14). A elastase, protease dos leucócitos polimorfonucleares, está envolvida na destruição crônica de tecidos associada à inflamação,<smiles>CC(CS)C(=O)N1CCCC1C(=O)O</smiles>

Captopril, 37<smiles>CC(NC(CCc1ccccc1)C(=O)O)C(=O)N1CCCC1C(=O)O</smiles>

Enalapril, 38<smiles>O=C(O)CN1C(=O)C(NC(CCc2ccccc2)C(=O)O)CCc2ccccc21</smiles>

Benazepril, 39<smiles>O=C(O)C(CCc1ccccc1)NC1CCCN2CCCC(C(=O)O)N2C1=O</smiles><smiles>O=C(O)C(CCc1ccccc1)NC1CCc2cccc3c2N(CC3C(=O)O)C(=O)C1</smiles>

Figura 11. Inibidores da enzima conversora da angiotensiva.

artrite e ao enfisema. Um pulmão normal está protegido da digestão proteolítica pela $\alpha_{1}$-antitripsina, um inibidor de protease endógeno, durante um processo inflamatório ${ }^{58}$. Os análogos restritos de $\mathbf{4 6}$ foram preparados ligando-se o carbono em posição $\beta$ da L-valina ao nitrogênio da glicina. Os valores do ângulo $\psi$ 


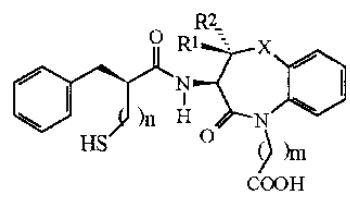

$42 \mathrm{X}=\mathrm{CH}_{2} ; \mathrm{S} ; \mathrm{O}$

Figura 12. Análogos restritos dos inibidores de metaloproteases ${ }^{52-54}$.

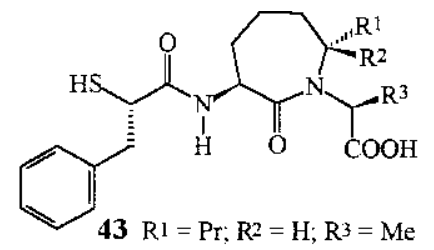<smiles>O=C(O)[C@H](S)Cc1ccccc1</smiles><smiles>CC(NC(=O)[C@@H](S)CC1C=CC=CC1)C(=O)N1CCCC1C(=O)O</smiles>

Figura 13. Análogos azepinônicos 43 e 44, inibidores de metaloproteases 55 .<smiles>[R]NC(C)(C)C(=O)N(CC(=O)NC(C(C)=O)C(C)C)C1Cc2ccccc2C1</smiles><smiles>[R]N([Tl])[C@H]1CCCN(CC(=O)N[C@@H](C(=O)C(F)(F)F)C(C)C)C1=O</smiles>

$47 \mathrm{n}=0,1,2$<smiles>[R]N([R])C1C[S@@](=O)[C@H]2CC=CC=C2N(CC(=O)NC(C(=O)C(F)(F)F)C(C)C)C1=O</smiles>

\section{$\mathrm{R}=p-\left[p-\mathrm{Cl}\left(\mathrm{C}_{6} \mathrm{H}_{4}\right) \mathrm{SO}_{2} \mathrm{NHCO}\right]\left(\mathrm{C}_{6} \mathrm{H}_{4}\right) \mathrm{CO}$}

Figura 14. Inibidores da elastase de leucócitos humanos ${ }^{57}$.

são obtidos em função do tamanho do ciclo lactâmico. Os resultados dos ensaios biológicos mostraram que os análogos monocíclicos do tipo $\mathbf{4 7}$ não têm atividade inibidora da elastase in vitro. Por sua vez, o análogo 48 possui atividade tanto in vitro como in vivo.

\section{Inibidores da trombina}

O grupo de proteases serínicas inclui as enzimas trombina, quimiotripsina, tripsina e subtilisina. A trombina é vista como a protease terminal chave da cascata de coagulação, reguladora da hemostase normal e do desenvolvimento de trombo intravascular anormal ${ }^{36,59}$. Além disto, a trombina estimula a agregação plaquetária e catalisa a conversão do fibrinogênio à fibrina ${ }^{60}$. Os análogos restritos (49, Figura 15) de inibidores da trombina foram obtidos pela incorporação de um monociclo lactâmico de maneira a mimetizar dobras $\beta$ dos tipos II e II', conformações dos inibidores 50 e $\mathbf{5 1}$. Os compostos do tipo 49 mostraram um aumento da atividade e da seletividade inibidora de protease em relação às referências $\mathbf{5 0}$ e $\mathbf{5 1}^{61}$.
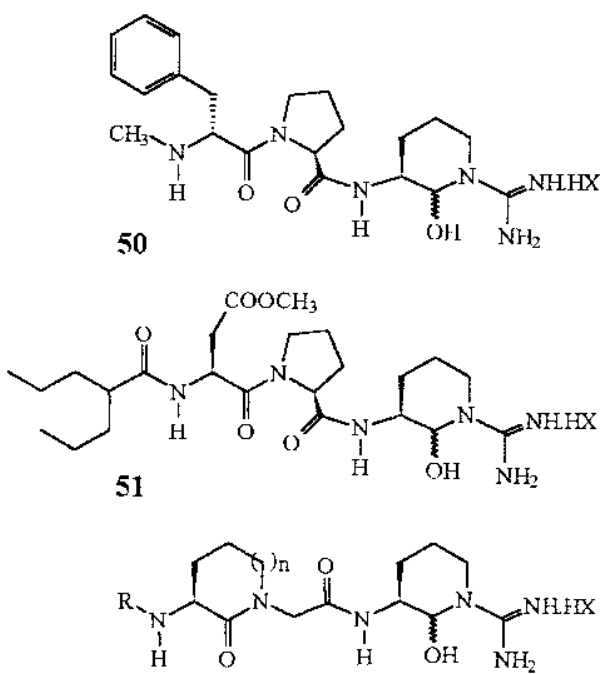

$49 \mathrm{R}=\mathrm{BnSO}_{2} ; \mathrm{Bn}(2-\mathrm{COOMe}) \mathrm{SO}_{2}$ $\mathrm{n}=1 ; 2$

Figura 15. Inibidores da trombina ${ }^{61}$.

\section{Oncogenes}

Proteínas da família Ras controlam o crescimento e a diferenciação celular. Quando as proteínas Ras estão inativas, as células não respondem aos fatores de crescimento que agem via receptores da tirosinaquinase ${ }^{36}$. As proteínas Ras podem ser reguladas tanto pelas proteínas de ativação de GTPase quanto por seu homólogo oncogênico. Deste modo, a ativação das proteínas Ras parece estar ligada a uma variedade de cânceres ${ }^{62}$.

Uma série de derivados azalactâmicos foi desenvolvida pela incorporação de unidades restritoras do tipo benzodiazepínico para mimetizar uma dobra dipeptídica natural de cadeias peptídicas inibidoras da CAAX farnesiltransferase ${ }^{63}$. A enzima utiliza o pirofosfato de farnesila como doador de um grupo farnesila, ligando-o ao resíduo cisteína da posição 4 da porção C-terminal de várias proteínas, dentre as quais as quatro proteínas $\operatorname{Ras}^{36,63 a}$. As porções C-terminais dessas proteínas possuem a seqüência CAAX, onde $\mathbf{C}$ corresponde a uma cisteína, $\mathbf{A}$ a dois aminoácidos com cadeia lateral alifática e $\mathbf{X}$ a uma metionina ou a uma serina ${ }^{63 a}$. A prevenção da farnesilação, pela mutação da seqüência CAAX ou pelo bloqueio da síntese do pirofosfato de farnesila, impede a capacidade de fixação das proteínas Ras oncogênicas à superfície da membrana plasmática ${ }^{63 a}$.

Tetrapeptídeos como Cys-Val-Phe-Met (52, Figura 16), onde um dos resíduos alifáticos é substituído por um resíduo aromático em posição 3 da seqüência CAAX, são inibidores da farnesiltransferase sem serem farnesilados. Infelizmente, quando em contato com células intactas, esses peptídeos tornam-se inativos ou 
são rapidamente degradados. Mimetizando os peptídeos do tipo 52, os análogos benzodiazepínicos $\mathbf{5 3 - 5 8}{ }^{63}$ foram preparados para limitar a metabolização. Os estudos farmacológicos mostraram que esses derivados são potentes inibidores da farnesiltransferase in vivo, podendo servir ao desenvolvimento de uma terapêutica de tumores do cólon, pulmão e pâncreas, nos quais as proteínas Ras oncogênicas contribuem ao crescimento celular anormal.

\section{Cys-Val-Phe-Met}

52<smiles>[X]C(=O)CN1C(=O)C(N([R])C(N)(F)C(F)(F)F)N=C(c2ccccc2)c2ccccc21</smiles>

$$
\begin{array}{lll}
\mathbf{5 3} & \mathrm{R}=\mathrm{H}_{;} & \mathrm{X}=\text { Met } \\
\mathbf{5 4} & \mathrm{R}=\mathrm{CH}_{3} ; & \mathrm{X}=\text { Met } \\
55 & \mathrm{R}=\mathrm{CH}_{3} ; & \mathrm{X}=\mathrm{Scr} \\
\mathbf{5 6} & \mathrm{R}=\mathrm{CH}_{3} ; & \mathrm{X}=\mathrm{Leu} \\
57 & \mathrm{R}=\mathrm{CH}_{3} ; & \mathrm{X}=\text { MetOCH } \\
\mathbf{5 8} & \mathrm{R}=\mathrm{CH}_{3} ; & \mathrm{X}=\text { MetNH }_{2}
\end{array}
$$

Figura 16. Inibidores da farnesiltransferase ${ }^{63}$.

Nos últimos anos, estudos da relação estrutura - atividade de análogos peptídicos farnesilados foram realizados. Os fatores a e $\alpha$, peptídeos feromônicos do Saccharomyces cerevisae, são ligantes funcionalmente equivalentes dos respectivos receptores da proteína $G^{64}$. A diferença estrutural mais significativa do fator a (59, Figura 17) em relação ao fator $\alpha$ é a farnesilação do resíduo cisteína. Dois análogos restritos 60 e $\mathbf{6 1}$ foram preparados, substituindo-se os resíduos $\mathrm{Lys}^{4}-\mathrm{Gly}^{5}$ e $\mathrm{Gly}^{5}-\mathrm{Val}^{6}$ da seqüência de 59 por unidades $\gamma$-lactâmicas ${ }^{64 a}$. As lactamas foram incorporadas de maneira a ligar o carbono $\alpha_{i}$ ao nitrogênio $\mathrm{N}_{i+1}$ dos resíduos 4-5 ou 5-6. A utilização de lactamas de configuração $R$ (em 60) e $S$ (em 61) foi proposta afim de mimetizar dobras $\beta$ II e $\beta$ II', centradas nas posições 4-5 ou 5-6, respectivamente. Os ensaios farmacológicos mostraram que o derivado $\mathbf{6 0}$ é um agonista efetivo do fator a, permitindo evidenciar uma conformação do peptídeo 59 em dobra $\beta$ II, centrada no fragmento Lys ${ }^{4}-G_{l y}^{5}$.

\section{Controle da função tireoidiana}

A partir de estudos de modelagem molecular, uma série de análogos do hormônio de liberação da tirotropina (TRH) foi preparada $^{65}$. O TRH 62 (Figura 18), um tripeptídeo hipotalâmico, interage com seu receptor (TRH-R), pertencente à família dos receptores da proteína $G^{66}$. Os análogos 63 e 64 foram preparados pela incorporação de uma lactama bicíclica unindo os átomos $\mathrm{C}_{\beta i}, \mathrm{~N}_{i+1}$ e $\mathrm{C}_{\beta i+1}$ do tripeptídeo. A síntese de análogos restritos com o resíduo de histidina em posição 2 não foi possível, o que levou à utilização do derivado $\mathbf{6 5}$ como referência nos estudos da relação estrutura - atividade da liberação do hormônio tiróide estimulante, controlada pela complexação TRH/THR-R ${ }^{65}$. O análogo $\mathbf{6 4}$, possuindo a unidade restritora de configuração $R$, mostrou maior afinidade pelo TRH-R que o análogo não restrito $\mathbf{6 5}$.<smiles></smiles><smiles>NC(=O)[C@@H]1CC[C@]2(I)CC(C3CCCC3)=C(N([Tl])C(=O)C3CCC(=O)N3)C(=O)N12</smiles><smiles>NC(=O)[C@@H]1CCCN1C(=O)[C@H](CC1CCCC1)NC(=O)C1CCC(=O)N1</smiles>

Figura 18. Incorporação de uma lactama 6,5-bicíclica nos análogos do $T R H^{65}$.

\section{Peptídeos ativos nos receptores das encefalinas}

Nos últimos anos, estudos de inclusão de 2-oxopiperazinas em sequiências peptídicas foram realizados para produzir análogos dos peptídeos ativos nos receptores das encefalinas ${ }^{67,68}$. Existem no mínimo três tipos distintos de receptores opióides através dos quais os analgésicos exercem seus efeitos biológicos ${ }^{59}$. Estes receptores foram denominados $\mu, \delta$ e

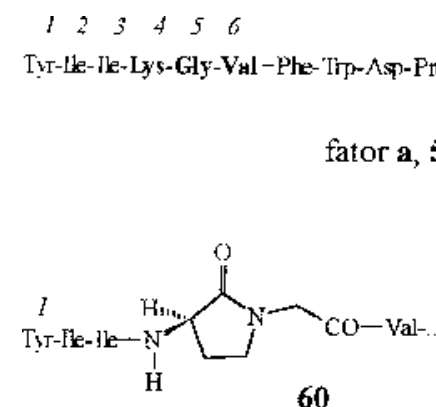

60

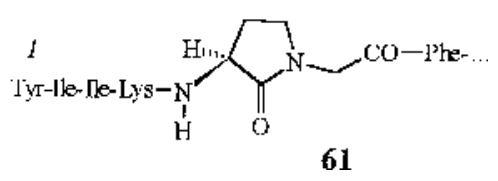

Figura 17. Análogos restritos do fator $\boldsymbol{a}^{64 a}$. 
$\kappa$. Os alcalóides da família da morfina ligam-se preferencialmente aos receptores $\mu$. As encefalinas, neuropeptídeos endógenos, mostram atividade principalmente nos receptores $\delta$, enquanto que seu precursor, a $\beta$-endorfina, apresenta maior atividade nos receptores $\mu$. Outro peptídeo, a dinorfina, tem atividade nos receptores $\kappa$. A dinorfina possui 17 aminoácidos na sua seqüência, cuja porção $\mathrm{N}$-terminal contém a seqüência da Leu-encefalina ${ }^{59}$. Ultimamente, análogos restritos da dinorfina foram preparados com o objetivo de caracterizar a relação estrutura - conformação e atividade da dinorfina $\mathrm{A}$ sobre os receptores $\kappa^{69}$. O fragmento 60-65 da $\beta$-endorfina corresponde a Met-encefalina ${ }^{59}$. Os compostos do tipo 66 e 67 (Figura 19) foram preparados com o intuito de se testar sua atividade opióide ${ }^{67}$. A série $\mathbf{6 6}$ foi obtida, utilizando-se a unidade dipeptídica restrita $\mathbf{6 8}$ incorporada na porção C-terminal das Leu- e Met-encefalinas (69 e 70). Os derivados da série 67 são os retro-análogos correspondentes da série $\mathbf{6 6}$.

$$
\text { Tyr-Gly-Gly-Phe-LeuOH }
$$

69
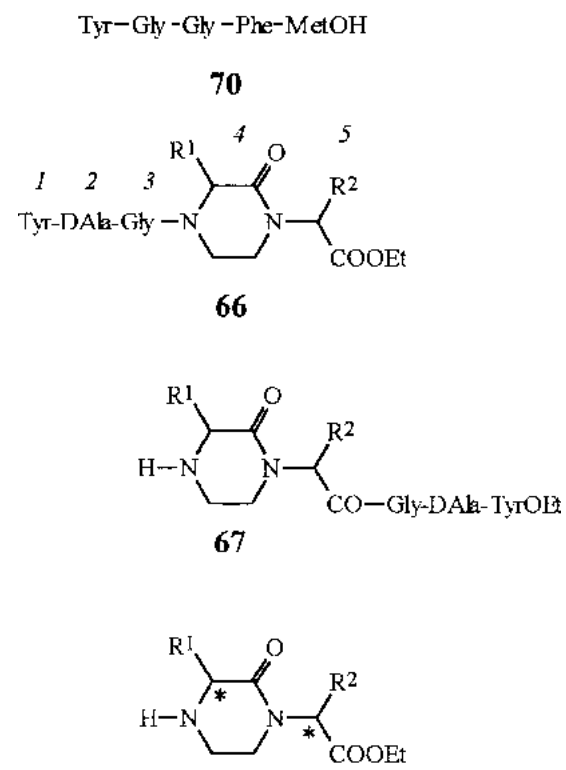

$$
\begin{gathered}
68 *=R ; S \\
\mathrm{R} 1 ; \mathrm{R}^{2}=\mathrm{PhCH}_{2} ; \mathrm{MeSCl}_{2} ;-\mathrm{Bu}
\end{gathered}
$$

Figura 19. Encefalinas e análogos restritos ${ }^{67}$.

Segundo os testes farmacológicos, a série $\mathbf{6 6}$ apresentou atividade opióide, no entanto mostrou baixa afinidade de ligação aos receptores em relação à Leu-encefalina. Adicionalmente, os ensaios mostraram que as configurações das posições 4 e 5 e as substituições $\mathrm{Met}^{5}$ ou Leu ${ }^{5}$ nos análogos 66 não influenciaram sua afinidade pelos receptores. Os retroanálogos (67) não apresentaram afinidade pelos receptores, o que confirmou a necessidade de um grupamento fenólico em posição 1 dos peptídeos para o planejamento estrutural de agonistas opióides.

Mais recentemente, os dipeptídeos restritos do tipo 68 (Figura 20) foram inseridos em seqüências tetrapeptídicas homólogas da dermofina $\mathbf{7 1}^{68}$, um heptapeptídeo opióide. Os homólogos 72 e 73 apresentaram atividade opióide. Seus análogos restritos (74) foram preparados unindo por uma ponte etilênica os átomos $\mathrm{N}_{3}$ e $\mathrm{N}_{4}$ dos compostos 72 e 73. Os análogos do tipo $\mathbf{7 4}$ apresentaram uma atividade opióide comparável à série 66.

\section{Tyr-DAla-Phe-Gly-Tyr-Pro-SerNH}

Dermofina, 71

\section{Tyr-DAla-Phe-Gly-NHz $\quad$ Tyr-DAla-Phe-Phe- $\mathrm{NH}_{2}$} 72

\section{3}

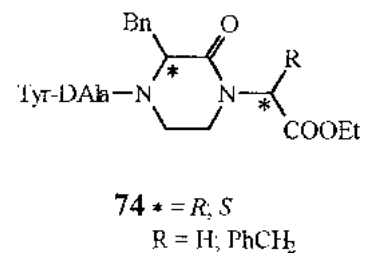

Figura 20. Dermofina 71, homólogos 72, 73 e análogos restritos ${ }^{68}$.

\section{PERSPECTIVAS}

O conhecimento das relações entre a estrutura, a função e a conformação de peptídeos e proteínas naturais está em constante desenvolvimento, mostrando a importância destas moléculas, e ainda, de enzimas e receptores de proteínas nos fenômenos fisiológicos que sustentam os processos biológicos. Várias unidades de restrição conformacional do tipo (aza)lactâmico foram preparadas e incorporadas em seqüências peptídicas para estudar ou buscar a atividade farmacológica e/ou a seletividade a um determinado receptor ou enzima, ainda que nem sempre tenha sido possível atingir estes objetivos. A abundância da utilização de restritores lactâmicos em relação aos azalactâmicos está diretamente ligada à disponibilidade de métodos de preparação dos primeiros. Os métodos de síntese assimétrica de unidades de restrição azalactâmicas são limitados. Recentemente, desenvolvemos um método de síntese assimétrica para obter unidades peptídicas azalactâmicas, no qual a etapa chave do processo é baseada na alquilação diastereosseletiva da posição C-3 de uma 2-oxopiperazina ${ }^{70}$. A maleabilidade na substituição do ciclo pode levar à utilização do método como uma ferramenta para estudos de relação estrutura - atividade de diferentes análogos oxopiperazínicos de seqüências ativas. Compreender as interações de estruturas peptídicas a um nível molecular é atualmente indispensável para o desenvolvimento de novos fármacos. Neste sentido, o desenvolvimento de moléculas ativas requer características estruturais que as tornem metabolicamente estáveis com a manutenção de suas atividades biológicas. Os avanços dos estudos das interações de ligantes ou substratos peptídicos de receptores ou enzimas se dão pela obtenção de peptideomiméticos, análogos não-peptídicos, ou ainda pela utilização de novos métodos de obtenção de fármacos, como por exemplo a química combinatória ${ }^{71}$. Esta estratégia leva à produção de coleções de moléculas diferentes preparadas por síntese ou biossíntese e testadas quanto suas atividades biológicas, acelerando o processo de obtenção de um novo fármaco ${ }^{72}$.

\section{AGRADECIMENTOS}

À CAPES/COFECUB pela concessão de bolsa de doutorado a A. P. na Université René Descartes - Paris V.

\section{REFERÊNCIAS}

1. Gante, J.; Angew. Chem. Int. Ed. Engl. 1994, 33, 1699.

2. Liskamp, R. M. J.; Rec. Trav. Chim. Pays-Bas 1994, 113,1 .

3. Fairlie, D. P.; Abbenante, G.; March, D. R.; Curr. Med. Chem. 1995, 2, 654. 
4. Marshall, G. R.; Tetrahedron 1993, 49, 3547.

5. Rose, G. D.; Gierasch, L. M.; Smith, J. A.; Adv. Protein Chem. 1985, 37, 1.

6. Kessler, H.; Angew. Chem. Int. Ed. Engl. 1982, $21,512$.

7. Hruby, V. J.; Life Sci. 1982, 31, 189.

8. Hruby, V. J.; Al-Obeidi, F.; Kazmierski, W.; Bio. Chem. J. 1990, 268, 249.

9. a) Freidinger, R. M.; Veber, D. F.; Schwenk Perlow, D.; Brooks, J. R.; Saperstein, R.; Science 1980, 210, 656. b) Freidinger, R. M.; Schwenk Perlow, D.; Veber, D. F.; J. Org. Chem. 1982, 47, 104. c) Blommaert, A. G. S.; Dhôtel, H.; Ducos, B.; Durieux, C.; Goudreau, N.; Bado, A.; Garbay, C.; Roques, B. P.; J. Med. Chem. 1997, 40, 647. d) Hruby, V. J.; Lu, D.; Sharma, S. D.; Castrucci, A. L.; Kesterson, R. A.; Al-Obeidi, F. A.; Hadley, M. E.; Cone, R. D.; J. Med. Chem. 1995, 38, 3454. e) Luo, P.; Braddock, D. T.; Subramanian, R. M.; Meredith, S. C.; Lynn, D. G.; Biochemistry 1994, 33, 12367. f) Houston Jr, M. E.; Wallace, A.; Bianchi, E.; Pessi, A.; Hodges, R. S.; J. Mol. Biol. 1996, 262, 270. g) Neugebauer, W.; Gagnon, L.; Whitfield, J.; Willick, G. E.; Int. J. Peptide Protein Res. 1994, 43, 555. h) Barbier, J. -R.; Neugebauer, W.; Morley, P.; Ross, V.; Soska, M.; Whitfield, J. F.; Willick, G.; J. Med. Chem. 1997, 40, 1373. i) Cervini, L. A.; Donaldson, C. J.; Koerber, S. C.; Vale, W. W.; Rivier, J. E.; J. Med. Chem. 1998, 41, 717.

10. Hanessian, S.; McNaughton-Smith, G.; Lombart, H. -G.; Lubell, W. D.; Tetrahedron 1997, 53, 12789.

11. a) Ede, N. J.; Rae, I. D.; Hearn, M. T. W.; Tetrahedron Lett. 1990, 31, 6071. b) Higgins, K. A.; Thompson, P. E.; Hearn, M. T. W.; Int. J. Peptide Protein Res. 1996, 48, 1.

12. Johnson, R. L.; Rajakumar, G.; Yu, K. -L.; Mishra, R. K.; J. Med. Chem. 1986, 29, 2104.

13. Yu, K. -L.; Rajakumar, G.; Srivastava, L. K.; Mishra, R. K.; Johnson, R. L.; J. Med. Chem. 1988, 31, 1430.

14. Björkman, S.; Castensson, S.; Sievertsson, H.; J. Med. Chem. 1979, 22, 931.

15. Procházka, Z.; Lebl, M.; Barth, T.; Hlavácek, J.; Trka, A.; Budesínsky, M.; Jost, K.; Collect. Czech. Chem. Commun. 1984, 49, 642.

16. Sreenivasan, U.; Mishra, R. K.; Johnson, R. L.; J. Med. Chem. 1993, 36, 256.

17. Subasinghe, N. L.; Bontems, R. J.; McIntee, E.; Mishra, R. K.; Johnson, R. L.; J. Med. Chem. 1993, 36, 2356.

18. Lee, J. M.; Ritzmann, R. F.; Fields, J. Z.; Peptides 1984, 5,7 .

19. Walter, R.; Ritzmann, R. F.; Bhargava, H.; Flexner, L. B.; Proc. Natl. Acad. Sci. 1979, 76, 518.

20. Bhargava, H. N.; J. Pharmacol. Exp. Ther. 1981, 218, 404.

21. Prasad, C.; Peptides 1995, 16, 151.

22. D'Angeli, F.; Marchetti, P.; Rondanin, R.; Bertolasi, V.; J. Org. Chem. 1996, 61, 1252.

23. Nagai, U.; Sato, K.; Tetrahedron Lett. 1985, 26, 647.

24. Nagai, U.; Sato, K.; Nakamura, R.; Kato, R.; Tetrahedron 1993, 49, 3577.

25. Nagai, U.; Sato, K.; J. Chem. Soc. Perkin Trans. I 1986, 1231.

26. Sato, K.; Hotta, M.; Dong, M. -H.; Hu, H. -Y.; Taulene, J. P.; Goodman, M.; Nagay, U.; Ling, N.; Int. J. Peptide Protein Res. 1991, 38, 340.

27. Bach II, A. C.; Markwalder, J. A.; Ripka, W. C.; Int. J. Peptide Protein Res. 1991, 38, 314.

28. Aebi, J. D.; Guillaume, D.; Dunlap, B. E.; Rich, D. H.; J. Med. Chem. 1988, 31, 1805.

29. Lee, J. P.; Dunlap, B.; Rich, D. H.; Int. J. Peptide Protein Res. 1990, 35, 481.

30. Hinds, M. G.; Richards, N. G. J.; Robinson, J. A.; J. Chem. Soc., Chem. Commun. 1988, 1447.
31. Hinds, M. G.; Welsh, J. H.; Brennand, D. M.; Fisher, J.; Glennie, M. J.; Richards, N. G. J.; Turner, D. L.; Robinson, J. A.; J. Med. Chem. 1991, 34, 1777.

32. a) Logan. M; E.; Goswqmi, R.; Tomczuk, B. E.; Venepqlli, B. R.; Annu. Rep. Med. Chem. 1991, 26, 43. b) Tanabe, T.; Otani, H.; Bao, L.; Mikami, Y.; Yasukura, T.; Ninomiya, T.; Ogawa, R.; Inagaki, C.; Eur. J. Pharmacol. 1996, 299, 187.

33. Genin, M. J.; Johnson, R. L.; J. Am. Chem. Soc. 1992, $114,8778$.

34. Genin, M. J.; Ojala, W. H.; Gleason, W. B.; Johnson, R. L.; J. Org. Chem. 1993, 58, 2334.

35. a) Ward, P.; Ewan, G. B.; Jordan, C. C.; Ireland, S. J.; Hagan, R. M.; Brown, J. R.; J. Med. Chem. 1990, 33, 1848. b) Deal, M. J.; Hagan, R. M.; Ireland, S. J.; Jordan, C. C.; McElroy, A. B.; Porter, B.; Ross, B. C.; Stephens-Smith, M.; Ward, P.; J. Med. Chem. 1992, 35, 4195.

36. Genuth, S. M.; Koeppen, B. M.; Kutchai, H. C.; Murphy, R. A. Stanton, B. A.; Staub, N. C.; Willis, W. D.; Principles of Physiology; 2a Edição; Mosby-Year Book, St. Louis 1996.

37. Fobian, Y. M.; D'avignon, A.; Moeller, K. D.; Bioorg. Med. Chem. Lett. 1996, 6, 315.

38. Thaisrivongs, S.; Pals, D. T.; Turner, S. R.; Kroll, L. T.; J. Med. Chem. 1988, 31, 1369.

39. Williams, P. D.; Perlow, D. S.; Payne, L. S.; Holloway, M. K.; Siegl, K. S.; Schorn, T. W.; Lynch, R. J.; Doyle, J. J.; Strouse, J. F.; Vlasuk, G. P.; Hoogsteen, K.; Springer, J. P.; Bush, B. L.; Halgren, T. A.; Richards, A. D.; Kay, J.; Veber, D. F.; J. Med. Chem. 1991, 34, 887.

40. Thomas, W. A.; Whitcombe, I. W. A.; J. Chem. Soc. Perkin Trans. II 1986, 747.

41. Attwood, M. R.; Hassal, C. H.; Kröhn, A.; Lawton, G.; Redshaw, S.; J. Chem. Soc. Perkin Trans. I 1986, 1011.

42. Thorsett, E. D.; Actual. Chim. Thér. 1986, 13, 257.

43. Thorsett, E. D.; Harris, E. E.; Aster, S. D.; Peterson, E. R.; Snyder, J. P.; Springer, J. P.; Hirshfield, J.; Tristram, E. W.; Patchett, A. A.; Ulm, E. H.; Vassil, T. C.; J. Med. Chem. 1986, 29, 251.

44. Yanagisawa, H.; Ishiara, S.; Ando, A.; Kanazaki, T.; Miyamoto, S.; Koike, H.; Iijima, Y.; Oizumi, K.; Matsushita, Y.; Hata, T.; J. Med. Chem. 1988, 31, 422.

45. Slade, J.; Stanton, J. L.; Ben-David, D.; Mazzenga, G. C.; J. Med. Chem. 1985, 28, 1517.

46. Gante, J.; Weitzel, R.; Tetrahedron Lett. 1988, 29, 181.

47. Watthey, J. W. H.; Stanton, J. L.; Desai, M.; Babiarz, J. E.; Finn, B. M.; J. Med. Chem. 1985, 28, 1511.

48. Flynn, G. A.; Giroux, E. L.; Dage, R. C.; J. Am. Chem. Soc. 1987, 109, 7914.

49. De Lombaert, S.; Blanchard, L.; Stamford, L. B.; Sperbeck, D. M.; Grim, M. D.; Jenson, T. M.; Rodriguez, H. R.; Tetrahedron Lett. 1994, 35, 7513.

50. Robl, J. A.; Karanewsky, D. S.; Assad, M. M.; Tetrahedron Lett. 1995, 36, 1593.

51. Burkholder, T. P.; Huber, E. W.; Flynn, G. A.; Bioorg. Med. Chem. Lett. 1993, 3, 231.

52. Robl, J. A.; Simpkins, L. M.; Stevenson, J.; Sun, C. -Q.; Murugesan, N.; Barrish, J. C.; Assad, M. M.; Bird, J. E.; Schaeffer, T. R.;Trippodo, N. C.; Petrillo, E. W.; Karaneswsky, D. S.; Bioorg. Med. Chem. Lett. 1994, 4, 1789.

53. Robl, J. A.; Simpkins, L. M.; Sulsky, R.; Sieber-Mcmaster, E.; Stevenson, J.; Kelly, Y. F.; Sun, C. -Q.; Misra, R. N.; Ryono, D. E.; Assad, M. M.; Bird, J. E.; Trippodo, N. C.; Karanewsky, D. S.; Bioorg. Med. Chem. Lett. 1994, 4, 1795.

54. a) Trippodo, N. C.; Robl, J. A.; Assad, M. M.; Bird, J. E.; Panchal, B. C.; Schaeffer, T. R.; Fox, M.; Giancarli, M. R.; Cheung, H. S.; J. Pharmacol. Exp. Ther. 1995, 275, 
745. b) Robl, J. A.; Sun, C. -Q.; Simpkins, L. M.; Ryono, D. E.; Barrish, J. C.; Karanewsky, D. S.; Assad, M. M.; Schaeffer, T. R.; Trippodo, N. C.; Bioorg. Med. Chem. Lett. 1994, 4, 2055.

55. Robl, J. A.; Cimarusti, M. P.; Simpkins, L. M.; Brown, B.; Ryono, D. E.; Bird, J. E.; Assad, M. M.; Schaeffer, T. R.; Trippodo, N. C.; J. Med. Chem. 1996, 39, 494.

56. Bohacek, R.; De Lombaert, S.; McMartin, C.; Priestle, J.; Grütter, M.; J. Am. Chem. Soc. 1996, 118, 8231.

57. Skiles, J. W.; Sorcek, R.; Jacober, S.; Miao, C.; Mui, P. W.; McNeil, D.; Rosenthal, A. S.; Bioorg. Med. Chem. Lett. 1993, 3, 773 .

58. Matthay, R. A.; Arroliga, A. C.; Cecil Textbook of Medicine; Ed. Bennett, J. C.; Plum, F.; 20ª Edição; W. B. Saunders Company, Philadelphia 1996.

59. Dugas, H., Bioorganic Chemistry: A Chemical Approach to Enzyme Action; $3^{\text {a }}$ Edição; Springer-Verlag, New York 1996.

60. a) Shuman, M.; Cecil Textbook of Medicine; Ed. Bennett, J. C.; Plum, F.; 20 a Edição; W. B. Saunders Company, Philadelphia 1996. b) Mosher, D. F.; Cecil Textbook of Medicine; Ed. Bennett, J. C.; Plum, F.; 20a Edição; W. B. Saunders Company, Philadelphia 1996.

61. Semple, J. E.; Rowley, D. C.; Brunck, T. K.; Ha-Uong, T.; Minami, N. K.; Owens, T. D.; Tamura, S. Y.; Goldmen, E. A.; Siev, D. V.; Ardecky, R. J.; Carpenter, S. H.; Ge, Y.; Richard, B. M.; Nolan, T. G.; Hakanson, K.; Tulinsky, A.; Nutt, R.; Ripka, W. C.; J. Med. Chem. 1996, 39, 4531.

62. Liu, E. T.; Cecil Textbook of Medicine; Ed. Bennett, J. C.; Plum, F.; 20a Edição; W. B. Saunders Company, Philadelphia 1996.

63. a) James, G. L.; Goldstein, J. L.; Brown, M. S.; Rawson, T. E.; Somers, T. C.; McDowell, R. S.; Crowley, C. W.; Lucas, B. K.; Levinson, A. D.; Marsters Jr, J. C.; Science 1993, 260, 1937. b) Leftheris, K.; Kline, T.; Natarajan, S.; De Virgilio, M. K.; Cho, Y. H.; Pluscec, J.; Ricca, C.; Robinson, S.; Seizinger, B. R.; Manne, V.; Meyers, C. A.; Bioorg. Med. Chem. Lett. 1994, 4, 887. c) Waldmann, H.; Schelhaas, M.; Nägele, E.; Kuhlmann, J.; Wittinghofer, A.; Schroeder, H.; Silvius, J. R.; Angew. Chem. Int. Ed. Engl. 1997, 36, 2238.
64. a) Zhang, Y. L.; Dawe, A. L.; Jiang, Y.; Becker, J. M.; Naider, F.; Biochem. Biophys. Res. Commun. 1996, 224, 327. b) Yang, W.; McKinney, A.; Becker, J. M.; Naider, F.; Biochemistry 1995, 34, 1308. c) RAo, M. H.; Yang, W.; Joshua, H.; Becker, J. M.; Naider, F.; Int. J. Peptide Protein Res. 1995, 45, 418.

65. a) Li, W.; Hanau, C. E.; D'avignon, A.; Moeller, K. D.; J. Org. Chem. 1995, 60, 8155. b) Beal, L. M.; Moeller, K. D.; Tetrahedron Lett. 1998, 39, 4639. c) Laakkonien, L.; Li, W.; Perlman, J. H.; Guernieri, F.; Osman, R.; Moeller, K. D.; Gershengorn, M. C.; Mol. Pharmacol. 1996, 49, 1092. d) Li, W.; Moeller, K. D.; J. Am. Chem. Soc. 1996, 118, 10106.

66. Revest, P.; Longstaff, A.; Molecular Neuroscience; Bios Scientific Publishers Ltd and Springer - Verlag, New York 1998.

67. Takenaka, H.; Miyake, H.; Kojima, Y.; Yasuda, M.; Gemba, M.; Yasuda, M.; J. Chem. Soc. Perkin Trans. I 1993, 933.

68. Yamashita, T.; Hatamoto, E.; Takenaka, H.; Kojima, Y.; Inoue, Y.; Gemba, M.; Yasuda, M.; Chem. Pharm. Bull. 1996, 44, 856 .

69. a) Lung, F. -D. T. Collins, N.; Stropova, D.; Davis, P.; Yamamura, H. I.; Porreca, F.; Hruby, V. J.; J. Med. Chem. 1996, 39, 1136. b) Arttamangkul, S.; Ishmael, J. E.; Murray, T. F.; Grandy, D. K.; DeLander, G. E.; Kieffer, B. L.; Aldrich, J. V.; J. Med. Chem. 1997, 40, 1211.

70. a) Pohlmann, A.; Schanen, V.; Guillaume, D.; Quirion, J. C.; Husson, H. -P.; J. Org. Chem. 1997, 62, 1016.b) Pohlmann, A.; Guillaume, D.; Quirion, J. -C.; Husson, H. P.; Tetrahedron Lett. 1997, 38, 5809. c) Pohlmann, A.; Guillaume, D.; Quirion, J. -C.; Husson, H. -P.; J. Peptide Res. 1998, 51, 116.

71. Dutton, F. E.; Lee, B. H.; Tetrahedron Lett. 1998, 39, 5313.

72. a) Gallop, M. A.; Barrett, R. W.; Dower, W. J.; Fodor, S. P. A.; Gordon, E. M.; J. Med. Chem. 1994, 37, 1233. b) Gordon, E. M.; Barrett, R. W.; Dower, W. J.; Fodor, S. P. A.; Gallop, M. A.; J. Med. Chem. 1994, 37, 1385. 